\title{
Unified description of quark and lepton mass matrices in a universal seesaw model
}

\author{
Yoshio Koide* \\ Department of Physics, University of Shizuoka, 52-1 Yada, Shizuoka 422-8526, Japan \\ Hideo Fusaoka ${ }^{\dagger}$ \\ Department of Physics, Aichi Medical University, Nagakute, Aichi 480-1195, Japan
}

(Received 13 September 2002; published 20 December 2002)

\begin{abstract}
In the democratic universal seesaw model, the mass matrices are given by $\bar{f}_{L} m_{L} F_{R}+\bar{F}_{L} m_{R} f_{R}+\bar{F}_{L} M_{F} F_{R}(f$ : quarks and leptons; $F$ : hypothetical heavy fermions), $m_{L}$ and $m_{R}$ are universal for up and down fermions, and $M_{F}$ has the structure $\left(1+b_{f} X\right)\left(b_{f}\right.$ is a flavor-dependent parameter, and $X$ is a democratic matrix). The model can successfully explain the quark masses and Cabibbo-Kobayashi-Maskawa mixing parameters in terms of the charged lepton masses by adjusting only one parameter $b_{f}$. However, so far, the model has not been able to give the observed bimaximal mixing for the neutrino sector. In the present paper, we consider that $M_{F}$ in the quark sectors are still "fully" democratic, while $M_{F}$ in the lepton sectors are partially democratic. Then the revised model can reasonably give a nearly bimaximal mixing without spoiling the previous success in the quark sectors.
\end{abstract}

DOI: 10.1103/PhysRevD.66.113004

PACS number(s): 14.60.Pq, 11.30.Hv, 12.15.Ff

\section{INTRODUCTION}

\section{A. What is the universal seesaw model?}

Stimulated by the recent progress of neutrino experiments, there has been considerable interest in a unified description of the quark and lepton mass matrices. One such unified model, a nonstandard model, the so-called "universal seesaw model" (USM) [1], is well known. The model describes not only the neutrino mass matrix $M_{\nu}$ but also the quark mass matrices $M_{u}$ and $M_{d}$ and the charged lepton mass matrix $M_{e}$ by seesaw-type matrices, universally: the model has hypothetical fermions $F_{i}(F=U, D, N, E ; i$ $=1,2,3$ ) in addition to the conventional quarks and leptons $f_{i}(f=u, d, \nu, e ; i=1,2,3)$, and these fermions are assigned to $f_{L}=(2,1), f_{R}=(1,2), \quad F_{L}=(1,1), \quad$ and $F_{R}=(1,1)$ of $\mathrm{SU}(2)_{L} \times \mathrm{SU}(2)_{R}$. The $6 \times 6$ mass matrix that is sandwiched between the fields $\left(\bar{f}_{L}, \bar{F}_{L}\right)$ and $\left(f_{R}, F_{R}\right)$ is given by

$$
M^{6 \times 6}=\left(\begin{array}{cc}
0 & m_{L} \\
m_{R} & M_{F}
\end{array}\right) \text {, }
$$

where $m_{L}$ and $m_{R}$ are universal for all the fermion sectors $(f=u, d, \nu, e)$ and only $M_{F}$ have structures dependent on the fermion sectors $F=U, D, N, E$. For $\Lambda_{L}<\Lambda_{R} \ll \Lambda_{S}$, where $\Lambda_{L}=O\left(m_{L}\right), \Lambda_{R}=O\left(m_{R}\right)$, and $\Lambda_{S}=O\left(M_{F}\right)$, the $3 \times 3$ mass matrix $M_{f}$ for the fermions $f$ is given by the well-known seesaw expression

$$
M_{f} \simeq-m_{L} M_{F}^{-1} m_{R} .
$$

Thus, the model answers the question why the masses of quarks (except for the top quark) and charged leptons are so small with respect to the electroweak scale $\Lambda_{L}$

\footnotetext{
*On leave at CERN, Geneva, Switzerland. Email address: yoshio.koide@cern.ch; koide@u-shizuoka-ken.ac.jp

${ }^{\dagger}$ Email address: fusaoka@aichi-med-u.ac.jp
}

$\left(\sim 10^{2} \mathrm{GeV}\right)$. On the other hand, the top quark mass enhancement is understood from the additional condition $\operatorname{det} M_{F}=0$ for the up-quark sector $(F=U)$ [2-4]. Since the seesaw mechanism does not work for the third family fermions, the top quark has a mass of the order of $m_{L} \sim \Lambda_{L}$.

For the neutrino sector, the mass matrix is given as

$$
\left(\bar{\nu}_{L} \bar{\nu}_{R}^{c} \bar{N}_{L} \bar{N}_{R}^{c}\right)\left(\begin{array}{cccc}
0 & 0 & 0 & m_{L} \\
0 & 0 & m_{R}^{T} & 0 \\
0 & m_{R} & M_{L} & M_{N} \\
m_{L}^{T} & 0 & M_{N}^{T} & M_{R}
\end{array}\right)\left(\begin{array}{c}
\nu_{L}^{c} \\
\nu_{R} \\
N_{L}^{c} \\
N_{R}
\end{array}\right),
$$

where $\nu_{R}^{c} \equiv\left(\nu_{R}\right)^{c} \equiv C \bar{\nu}_{R}^{T}$. Since $O\left(M_{N}\right) \sim O\left(M_{L}\right) \sim O\left(M_{R}\right)$ $\gg O\left(m_{R}\right) \gg O\left(m_{L}\right)$, we obtain the mass matrix $M_{\nu}$ for the active neutrinos $\nu_{L}$,

$$
M_{\nu} \simeq-m_{L} M_{R}^{-1} m_{L}^{T}
$$

If we take the ratio $O\left(m_{L}\right) / O\left(m_{R}\right)$ suitably small, we can understand the smallness of the observed neutrino masses reasonably.

For an embedding of the model into a grand unification scenario, for example, see Ref. [5], where the possibility of $\mathrm{SO}(10) \times \mathrm{SO}(10)$ was discussed.

\section{B. What is the democratic universal seesaw model?}

As an extended version of the USM, the "democratic" USM [2,3] is also well known. The model has successfully given the quark masses and the Cabibbo-KobayashiMaskawa (CKM) [6] matrix parameters in terms of the charged lepton masses. The outline of the model is as follows.

(i) The mass matrices $m_{L}$ and $m_{R}$ have the same structure, except for their phase factors 


$$
m_{L}^{f}=m_{R}^{f} / \kappa=m_{0} Z_{f},
$$

where $\kappa$ is a constant with $\kappa \gg 1$ and $Z_{f}$ are given by

$$
\begin{gathered}
Z_{f}=P\left(\delta_{f}\right) Z, \\
P\left(\delta_{f}\right)=\operatorname{diag}\left(e^{i \delta_{1}^{f}}, e^{i \delta_{2}^{f}}, e^{i \delta_{3}^{f}}\right), \\
Z=\operatorname{diag}\left(z_{1}, z_{2}, z_{3}\right),
\end{gathered}
$$

with $z_{1}^{2}+z_{2}^{2}+z_{3}^{2}=1$.

(ii) In the basis on which the matrices $m_{L}^{f}$ and $m_{R}^{f}$ are diagonal, the mass matrices $M_{F}$ are given by the form

$$
\begin{gathered}
M_{F}=m_{0} \lambda\left(\mathbf{1}+3 b_{f} X\right), \\
\mathbf{1}=\left(\begin{array}{lll}
1 & 0 & 0 \\
0 & 1 & 0 \\
0 & 0 & 1
\end{array}\right), \quad X=\frac{1}{3}\left(\begin{array}{ccc}
1 & 1 & 1 \\
1 & 1 & 1 \\
1 & 1 & 1
\end{array}\right) .
\end{gathered}
$$

(iii) The parameter $b_{f}$ for the charged lepton sector is given by $b_{e}=0$, so that in the limit of $\kappa / \lambda \ll 1$ the parameters $z_{i}$ are given by

$$
\frac{z_{1}}{\sqrt{m_{e}}}=\frac{z_{2}}{\sqrt{m_{\mu}}}=\frac{z_{3}}{\sqrt{m_{\tau}}}=\frac{1}{\sqrt{m_{e}+m_{\mu}+m_{\tau}}} .
$$

Then the up- and down-quark masses are successfully given $[2,3]$ by the choice of $b_{u}=-1 / 3$ and $b_{d}=-e^{i \beta_{d}}\left(\beta_{d}\right.$ $\left.=18^{\circ}\right)$, respectively. Here, note that the choice $b_{u}=-1 / 3$ gives $\operatorname{det} M_{U}=0$, so that the case with $b_{u}=-1 / 3$ gives $m_{t}$ $\sim O\left(m_{L}\right)$. Another motivation for the choice $b_{u}=-1 / 3$ is that the model with $b_{e}=0$ and $b_{u}=-1 / 3$ leads to the successful relation $[7,2] m_{u} / m_{c} \simeq(3 / 4)\left(m_{e} / m_{\mu}\right)$, which is almost independent of the value of the seesaw suppression factor $\kappa / \lambda$. For the choice of $b_{u}=-1 / 3$ and $b_{d}=$ $-e^{i \beta_{d}}\left(\beta_{d}=18^{\circ}\right)$, the CKM matrix parameters are successfully given $[2,3]$ by taking

$$
\delta_{1}^{u}-\delta_{1}^{d}=\delta_{2}^{u}-\delta_{2}^{d}=0, \quad \delta_{3}^{u}-\delta_{3}^{d} \simeq \pi .
$$

A more detailed formulation (including the renormalization group equation effects) is found in Ref. [8].

\section{What is the problem?}

It seems that the model is successful as far as the quark mass phenomenology is concerned, so that the future task is only to give a more reliable theoretical base to the model. However, the democratic USM has a serious problem in the neutrino phenomenology. In the previous model, the parameters $z_{i}$ are fixed by the observed charged lepton masses as shown in Eq. (1.11), and the only adjustable parameter is $b_{\nu}$ defined by Eq. (1.9). For $b_{\nu} \simeq-1 / 2\left(b_{\nu} \simeq-1\right)$, we can obtain the maximal mixing between $\nu_{\mu}$ and $\nu_{\tau}\left(\nu_{e}\right.$ and $\left.\nu_{\mu}\right)$ [9], while we cannot give the nearly bimaximal mixing that is suggested by the observed atmospheric $[10]$ and solar $[11,12]$ neutrino data.
This suggests that the previous model with the universal structure of $M_{F}$ is too tight. Therefore, in the next section, we assume that for the lepton sectors the democratic matrix $X$ in Eq. (1.9) will be changed by a "partially" democratic matrix, which is given by a rotation $R_{X}$ from the fully democratic matrix in the quark sector. Then we can obtain the observed nearly bimaximal mixing. However, generally speaking, the success is not so remarkable because we have three additional parameters in the rotation matrix $R_{X}$. The problem is whether the rotation $R_{X}$ has a physical meaning or not.

In Sec. II, we will investigate a rotation matrix $R_{X}$ that leads to the observed nearly bimaximal mixing and suggests an interesting relation between quarks and leptons. In Sec. III, the numerical results are given and neutrino phenomenology is discussed. In Sec. IV, the mysterious characteristics of the rotation matrix $R_{X}$ are discussed. Finally, Sec. V is devoted to the conclusions.

\section{II. $S_{2}$ SYMMETRY VERSUS $S_{3}$ SYMMETRY}

\section{A. Basic assumption}

For the quark sectors, the model is essentially unchanged from the previous model, i.e., the mass terms are given by

$$
\begin{aligned}
m_{0} \sum_{f=u, d}\left[\bar{f}_{L} Z F_{R}+\kappa \bar{F}_{L} Z f_{R}\right. \\
\left.\quad+\lambda \bar{F}_{L} P^{\dagger}\left(\delta_{f}\right)\left(\mathbf{1}+3 b_{f} X\right) P\left(\delta_{f}\right) F_{R}\right]+ \text { H.c. },
\end{aligned}
$$

where we have changed the place of the phase matrix $P$ from $Z$ to $M_{F}$, so that $m_{L}$ and $m_{R}$ are completely flavor independent. On this basis the mass matrices $m_{L}$ and $m_{R}$ are diagonal, and the mass matrix $M_{F}$ is invariant under the permutation symmetry $S_{3}$ except for the phase factors. As investigated in Refs. [2,3], in order to give reasonable values of the CKM matrix parameters, it was required to choose

$$
P\left(\delta_{u}\right) P^{\dagger}\left(\delta_{d}\right)=P\left(\delta_{u}-\delta_{d}\right) \simeq \operatorname{diag}(1,1,-1),
$$

although the origin of such a phase inversion is still an open question. In this paper, we assume

$$
P\left(\delta_{u}\right)=\operatorname{diag}(1,1,-1), \quad P\left(\delta_{d}\right)=\operatorname{diag}(1,1,1) .
$$

For the lepton sectors, we assume

$$
\begin{aligned}
m_{0} \sum_{f=e, \nu}\left[\bar{f}_{L} Z F_{R}+\kappa \bar{F}_{L} Z f_{R}\right. \\
\left.\quad+\lambda \bar{F}_{L}^{\prime} P^{\dagger}\left(\delta_{f}\right)\left(\mathbf{1}+3 b_{f} X\right) P\left(\delta_{f}\right) F_{R}^{\prime}\right]+ \text { H.c. },
\end{aligned}
$$

where, for convenience, we have dropped the Majorana mass terms $\bar{N}_{L} M_{L} N_{L}^{c}+\bar{N}_{R}^{c} M_{R} N_{R}$ from the expression (2.4), since we always assume that the Majorana mass matrices $M_{L}$ and $M_{R}$ have the same structure as the Dirac mass matrix $M_{N}$ $=\lambda m_{0} P^{\dagger}\left(\delta_{\nu}\right)\left(\mathbf{1}+3 b_{\nu} X\right) P\left(\delta_{\nu}\right)$. In Eq. (2.4), we have defined

$$
F^{\prime}=R_{X}^{T} F
$$


Here, we have tacitly assumed symmetries $\mathrm{SU}(2)_{L}^{\prime}$ $\times \mathrm{SU}(2)_{R}^{\prime}$ for the heavy fermions $F_{L}$ and $F_{R}$ in addition to the symmetries $\mathrm{SU}(2)_{L} \times \mathrm{SU}(2)_{R}$ for $f_{L}$ and $f_{R}$, so that we have required the same rotation $R_{X}$ for the heavy leptons $\left(N_{i}, E_{i}\right)_{L}$ [and $\left.\left(N_{i}, E_{i}\right)_{R}\right]$. Then the heavy lepton mass terms in Eq. (2.4) can be rewritten as

$$
m_{0} \lambda \sum_{f=e, \nu} \bar{F}_{L}\left(\mathbf{1}+3 b_{f} X_{f}\right) F_{R}+\text { H.c. }
$$

where

$$
X_{f}=R_{X} P^{\dagger}\left(\delta_{f}\right) X P\left(\delta_{f}\right) R_{X}^{T} .
$$

We take the phase matrices in the lepton sectors as

$$
\begin{gathered}
P\left(\delta_{\nu}\right)=P\left(\delta_{u}\right)=\operatorname{diag}(1,1,-1), \\
P\left(\delta_{e}\right)=P\left(\delta_{d}\right)=\operatorname{diag}(1,1,1),
\end{gathered}
$$

corresponding to Eq. (2.3). Then, the effective charged lepton and neutrino mass matrices are given by

$$
\begin{gathered}
M_{e} \simeq-m_{0} \frac{\kappa}{\lambda} Z R_{X}\left(\mathbf{1}+3 a_{e} X\right) R_{X}^{T} Z \equiv m_{0}^{e} Z\left(1+3 a_{e} X_{e}\right) Z \\
M_{\nu} \simeq-m_{0} \frac{1}{\lambda} Z R_{X} P^{\dagger}\left(\delta_{\nu}\right)\left(\mathbf{1}+3 a_{\nu} X\right) P\left(\delta_{\nu}\right) R_{X}^{T} Z \\
\equiv m_{0}^{\nu} Z\left(1+3 a_{\nu} X_{\nu}\right) Z
\end{gathered}
$$

where $m_{0}^{e}=-m_{0}(\kappa / \lambda), m_{0}^{\nu}=-m_{0} / \lambda, X_{e}=R_{X} X R_{X}^{T}$, and $X_{\nu}=R_{X} P^{\dagger}\left(\delta_{\nu}\right) X P\left(\delta_{\nu}\right) R_{X}^{T}$, and we have used

$$
\begin{gathered}
\left(\mathbf{1}+3 b_{f} X\right)^{-1}=\mathbf{1}+3 a_{f} X, \\
a_{f}=-b_{f} /\left(1+3 b_{f}\right) .
\end{gathered}
$$

The rotation $R_{X}$ is between the basis in the quark sectors and that in the lepton sectors. Our interests are as follows: What rotation $R_{X}$ can give reasonable neutrino masses and mixings? What relation does it suggest between quarks and leptons?

\section{B. A special form of $\boldsymbol{R}_{X}$}

In the heavy down-quark mass matrix $M_{D}$, we have considered that the matrix $X_{d}$ is completely democratic, i.e., $X_{d}=X$ defined by Eq. (1.10). Hereafter, we define the "fully" democratic matrix $X$ defined in Eq. (1.10) as $X_{3}$ $\equiv X$. The matrix $X_{f}$ is a rank-1 matrix, which satisfies the relation $\left(X_{f}\right)^{2}=X_{f}$. We suppose that the matrices $X_{f}(f$ $=e, \nu)$ in the heavy lepton sectors will not be "fully" democratic, but "partially" democratic. The simplest expression of the partially democratic matrix is

$$
X_{2} \equiv \frac{1}{2}\left(\begin{array}{lll}
1 & 1 & 0 \\
1 & 1 & 0 \\
0 & 0 & 0
\end{array}\right)
$$

We identify $X_{e}$ as $X_{e}=X_{2}$. The rotation $R_{X}$, which transforms $X_{3}$ into $X_{2}$, i.e.,

$$
R_{X} X_{3} R_{X}^{T}=X_{2}
$$

is given by

$$
R_{X}=R_{3}\left(-\frac{\pi}{4}\right) \cdot T \cdot R_{3}(\theta) \cdot\left(-P_{3}\right) \cdot A,
$$

$$
\begin{aligned}
& R_{3}(\theta)=\left(\begin{array}{ccc}
\cos \theta & \sin \theta & 0 \\
-\sin \theta & \cos \theta & 0 \\
0 & 0 & 1
\end{array}\right) \\
& \left.A=\mid \begin{array}{ccc}
\frac{1}{\sqrt{2}} & -\frac{1}{\sqrt{2}} & 0 \\
\frac{1}{\sqrt{6}} & \frac{1}{\sqrt{6}} & -\frac{2}{\sqrt{6}} \\
\frac{1}{\sqrt{3}} & \frac{1}{\sqrt{3}} & \frac{1}{\sqrt{3}}
\end{array}\right), \\
& T=\left(\begin{array}{ccc}
0 & 0 & 1 \\
0 & 1 & 0 \\
1 & 0 & 0
\end{array}\right), \quad P_{3}=\left(\begin{array}{ccc}
1 & 0 & 0 \\
0 & 1 & 0 \\
0 & 0 & -1
\end{array}\right)
\end{aligned}
$$

The matrix $A$ transforms the fully democratic matrix $X_{3}$ to the diagonal form

$$
A X_{3} A^{T}=\left(\begin{array}{lll}
0 & 0 & 0 \\
0 & 0 & 0 \\
0 & 0 & 1
\end{array}\right) \equiv Z_{3}
$$

The matrix $Z_{3}$ is invariant under the rotation $R_{3}(\theta)$ with an arbitrary $\theta$. The transformation $T$ has been introduced in order to transform $Z_{3}$ to $Z_{1} \equiv \operatorname{diag}(1,0,0)$. Finally, the rotation $R_{3}(-\pi / 4)$ transforms $Z_{1}$ to $X_{2}$. In the definition of $R_{X}$, [Eq. (2.15)] we have inserted the matrix $-P_{3}$ on the lefthand side of the matrix $A$. The matrix $-P_{3}$ does not have any effect on the matrix $Z_{3}$. In the numerical study in the next section, we are interested in the case where $\left(R_{X}\right)_{13}$ takes a small positive value, so that the matrix $-P_{3}$ has been introduced to make the numerical search easier.

For further convenience, we express the rotation $R_{3}(\theta)$ by a new angle parameter $\varepsilon=\theta-\pi / 4$. Then, the explicit form of $R_{X}$ is given by 


$$
R_{X}=\left(\begin{array}{ccc}
x_{3} & x_{2} & x_{1} \\
\sqrt{\frac{2}{3}}-x_{3} & \sqrt{\frac{2}{3}}-x_{2} & \sqrt{\frac{2}{3}}-x_{1} \\
\sqrt{\frac{2}{3}}\left(x_{1}-x_{2}\right) & \sqrt{\frac{2}{3}}\left(x_{3}-x_{1}\right) & \sqrt{\frac{2}{3}}\left(x_{2}-x_{3}\right)
\end{array}\right),
$$

where $x_{i}$ are given by

$$
\begin{gathered}
x_{1}=\frac{1}{\sqrt{6}}-\frac{c-s}{\sqrt{6}}, \\
x_{2}=\frac{1}{\sqrt{6}}+\frac{c-s}{2 \sqrt{6}}-\frac{c+s}{2 \sqrt{2}}, \\
x_{3}=\frac{1}{\sqrt{6}}+\frac{c-s}{2 \sqrt{6}}+\frac{c+s}{2 \sqrt{2}}
\end{gathered}
$$

$(s=\sin \varepsilon$ and $c=\cos \varepsilon)$ and they satisfy the relations

$$
\begin{gathered}
x_{1}^{2}+x_{2}^{2}+x_{3}^{2}=1, \\
x_{1}+x_{2}+x_{3}=\sqrt{\frac{3}{2}} .
\end{gathered}
$$

Since we have assumed the inversion $P\left(\delta_{u}\right)$ [Eq. (2.3)], the heavy up-quark mass matrix $M_{U}$ [and therefore the matrix $\left.P^{\dagger}\left(\delta_{u}\right) X_{3} P\left(\delta_{u}\right)\right]$ is not invariant under the permutation symmetry $\mathrm{S}_{3}$, although it is still invariant under the permutation symmetry $\mathrm{S}_{2}$ for the fields $u_{1}$ and $u_{2}$, because of the form

$$
X_{u}=P^{\dagger}\left(\delta_{u}\right) X_{3} P\left(\delta_{u}\right)=\frac{1}{3}\left(\begin{array}{ccc}
1 & 1 & -1 \\
1 & 1 & -1 \\
-1 & -1 & 1
\end{array}\right) \equiv X_{3}^{\prime} .
$$

Since the matrix $X_{3}^{\prime}$ is not invariant under the permutation symmetry $\mathrm{S}_{3}$, the neutral heavy lepton mass matrix $M_{N}$ has a somewhat complicated form: the rank-1 matrix $X_{\nu}$ is generally given by

$$
X_{\nu}=\left(\begin{array}{ccc}
y_{1}^{2} & y_{1} y_{2} & y_{1} y_{3} \\
y_{1} y_{2} & y_{2}^{2} & y_{2} y_{3} \\
y_{1} y_{3} & y_{2} y_{3} & y_{3}^{2}
\end{array}\right),
$$

where $y_{i}$ satisfy the normalization $y_{1}^{2}+y_{2}^{2}+y_{3}^{2}=1$. By comparing the result $R_{X} X_{3}^{\prime} R_{X}^{T}$ from Eq. (2.20) with the expression (2.25), we find

$$
y_{1}=\frac{1}{3 \sqrt{2}}+\frac{\sqrt{2}}{3}(c-s),
$$

$$
\begin{gathered}
y_{2}=\frac{1}{3 \sqrt{2}}-\frac{\sqrt{2}}{3}(c-s), \\
y_{3}=\frac{2}{3}(c+s) .
\end{gathered}
$$

In the next section, we will investigate the neutrino mass matrix (2.10) numerically. The expression (2.25) is not always $S_{2}$ invariant. Therefore, in the next section, we will require the matrix $X_{\nu}$ to also have an $\mathrm{S}_{2}$ invariant form. Then the parameter $\varepsilon$ is fixed, so that the model can again be reduced to a one-parameter model with only $b_{\nu}$.

\section{NUMERICAL STUDY OF THE NEUTRINO MASS MATRIX}

In order to find the numerical study of the neutrino mass matrix (2.10) without spoiling the previous success in the quark sectors, we evaluate Eq. (2.9) in the limit of $b_{e} \rightarrow 0$. Then the values of the parameters $z_{i}$ are still given by Eq. (1.11). Therefore, the numerical success in the quark sectors $[2,3]$ is unchanged. The matrix $U_{\nu}$ by which the mass matrix (2.10) is diagonalized as

$$
U_{\nu}^{\dagger} M_{\nu} U_{\nu}^{*}=D_{\nu} \equiv \operatorname{diag}\left(m_{1}^{\nu}, m_{2}^{\nu}, m_{3}^{\nu}\right)
$$

is the so-called Maki-Nakagawa-Sakata-Pontecorvo (MNSP) [13] matrix. Hereafter, we will simply call $U_{\nu}$ the lepton mixing matrix.

The neutrino mass matrix $M_{\nu}$ has two parameters $b_{\nu}$ and $\varepsilon$. First, we try to require that the matrix $X_{\nu}$ be invariant under a permutation symmetry $\mathrm{S}_{2}$. Although, as suggested from the form $X_{e}=X_{2}$ in Eq. (2.13), the case with $y_{1}=y_{2}$ is very interesting, regrettably it cannot give the observed nearly bimaximal mixing for any value of $b_{\nu}$. Of the possible cases $y_{1}=y_{2}, y_{2}=y_{3}$, and $y_{3}=y_{1}$, only the case $y_{3}$ $=y_{1}$ has a solution that gives reasonable mixing and mass values. The case with $y_{1}=y_{3}$ fixes the parameters $x_{i}$ and $\varepsilon$ as

$$
\begin{gathered}
y_{1}=y_{3}=0.6900, \quad y_{2}=-0.2186, \\
x_{1}=0.014811, \quad x_{2}=0.23904, \quad x_{3}=0.970890, \\
\varepsilon=2.043^{\circ} .
\end{gathered}
$$

As we defined in Eqs. (2.22) and (2.23), the parameters $x_{i}$ satisfy the relation

$$
x_{1}^{2}+x_{2}^{2}+x_{3}^{2}=\frac{2}{3}\left(x_{1}+x_{2}+x_{3}\right)^{2} .
$$

On the other hand, it is well known that the observed charged lepton masses satisfy the relation [14]

$$
m_{e}+m_{\mu}+m_{\tau}=\frac{2}{3}\left(\sqrt{m_{e}}+\sqrt{m_{\mu}}+\sqrt{m_{\tau}}\right)^{2},
$$

i.e., 
TABLE I. Predictions of the neutrino masses and mixing parameters. For the predictions $\Delta m_{i j}^{2}$ and $m_{i}^{\nu}$, we have used the value $\Delta m_{32}^{2}=2.5 \times 10^{-3} \mathrm{eV}^{2}$ from the atmospheric neutrino data [10]. In case $\mathrm{A}$, the values $x_{i}$ are determined from the requirement $y_{1}$ $=y_{3}$, and the values $z_{i}$ are obtained from the relation (3.7) and the observed values of $m_{e}$ and $m_{\mu}$. In case B, the values $x_{i}$ are taken as $x_{i}=z_{i}$, where $z_{i}$ are obtained as in case $\mathrm{A}$.

\begin{tabular}{lcc}
\hline \hline & Case A with $x_{i} \neq z_{i}$ & Case B with $x_{i}=z_{i}$ \\
\hline$b_{\nu}$ & -0.680 & -0.684 \\
$a_{\nu}$ & -0.654 & -0.650 \\
$m_{1}^{\nu}(\mathrm{eV})$ & $2.39 \times 10^{3}$ & $2.43 \times 10^{3}$ \\
$m_{2}^{\nu}(\mathrm{eV})$ & $7.46 \times 10^{3}$ & $7.48 \times 10^{3}$ \\
$m_{3}^{\nu}(\mathrm{eV})$ & $5.06 \times 10^{2}$ & $5.06 \times 10^{2}$ \\
$\Delta m_{21}^{2}\left(\mathrm{eV}^{2}\right)$ & $5.00 \times 10^{-5}$ & $5.01 \times 10^{-5}$ \\
$\Delta m_{32}^{2}\left(\mathrm{eV}^{2}\right)$ & $2.50 \times 10^{-3}$ & $2.50 \times 10^{-3}$ \\
$\Delta m_{21}^{2} / \Delta m_{32}^{2}$ & $2.00 \times 10^{-2}$ & $2.00 \times 10^{-2}$ \\
$\sin ^{2} 2 \theta_{12}$ & 0.796 & 0.801 \\
$\left(\tan ^{2} \theta_{12}\right)$ & $(0.377)$ & $(0.383)$ \\
$\sin ^{2} 2 \theta_{23}$ & 0.978 & 0.979 \\
$\left|\left(U_{\nu}\right)_{13}\right|^{2}$ & $6.65 \times 10^{-3}$ & $6.68 \times 10^{-3}$ \\
\hline \hline
\end{tabular}

$$
z_{1}^{2}+z_{2}^{2}+z_{3}^{2}=\frac{2}{3}\left(z_{1}+z_{2}+z_{3}\right)^{2}
$$

In fact, from relation (3.6), the observed charged lepton masses $m_{e}$ and $m_{\mu}$ predict $m_{\tau}^{\text {theor }}=1776.97 \mathrm{MeV}$, which is in excellent agreement with the observed value $m_{\tau}^{\text {obs }}$ $=1776.99_{-0.26}^{+0.29} \mathrm{MeV}$, together with the parameter values of $z_{i}$ for $b_{e}=0$

$$
z_{1}=0.016473, \quad z_{2}=0.23687, \quad z_{3}=0.97140,
$$

which correspond to

$$
\varepsilon=2.268^{\circ} \text {. }
$$

It should be noted that the values (3.3) [and (3.4)] are very near to the values (3.8) [and (3.9)]. We may consider that the parameters $z_{i}$ are identical with the $x_{i}$, which gives $y_{3}=y_{1}$ at a unification scale $\mu=M_{X}$.

In the numerical search, the value of the parameter $b_{\nu}$ is determined as the prediction $R=\Delta m_{21}^{2} / \Delta m_{32}^{2}$ gives the observed value $[10,12]$

$$
R_{o b s} \simeq \frac{5.0 \times 10^{-5} \mathrm{eV}^{2}}{2.5 \times 10^{-3} \mathrm{eV}^{2}}=2.0 \times 10^{-2}
$$

In Table I, we list the numerical results of $b_{\nu}, m_{i}^{\nu}, \Delta m_{21}^{2}$, $\Delta m_{32}^{2}, \sin ^{2} 2 \theta_{12}, \sin ^{2} 2 \theta_{23}$, and $\left|\left(U_{\nu}\right)_{13}\right|^{2}$ as case A. Here, for simplicity, we have used the values $4\left|\left(U_{\nu}\right)_{11}\right|^{2}\left|\left(U_{\nu}\right)_{12}\right|^{2}$ and $4\left|\left(U_{\nu}\right)_{23}\right|^{2}\left|\left(U_{\nu}\right)_{33}\right|^{2}$ as the values of $\sin ^{2} 2 \theta_{12}$ and $\sin ^{2} 2 \theta_{23}$, respectively, because $R \ll 1$. For reference, in Table I, we also list a case with $x_{i}=z_{i}=\sqrt{m_{i}^{e} /\left(m_{e}+m_{\mu}+m_{\tau}\right)}$ as case B. In this case, the scenario is that the partially democratic form of $X_{\nu}$ with $y_{3}=y_{1}$ is slightly broken at $\mu=m_{Z}$, still keeping $x_{i}=z_{i}$. From the numerical point of view, there is no essential difference between the two cases.

The predicted value of $\sin ^{2} 2 \theta_{12}\left(\tan ^{2} \theta_{12}\right)$

$$
\sin ^{2} 2 \theta_{12}=0.80 \quad\left(\tan ^{2} \theta_{12}=0.38\right)
$$

is in good agreement with the present best fit value [12] $\tan ^{2} \theta_{\text {solar }}=0.34\left(\sin ^{2} 2 \theta_{\text {solar }}=0.76\right)$. It should be noted that the predicted value (3.11) gives a suitable deviation from $\sin ^{2} 2 \theta_{12}=1.0$, although the Zee-type model cannot give such a sizable deviation from $\sin ^{2} 2 \theta_{12}=1.0$ [15].

It is also worth noting that in Table I the value of $b_{\nu}$ is very near to $b_{\nu}=-2 / 3$. The results $b_{e}=0, b_{u}=-1 / 3, b_{\nu}$ $\simeq-2 / 3$, and $b_{d} \simeq-1$ may suggest the existence of some unified rule for $b_{f}$.

Finally, we must excuse ourselves for taking the parameter $b_{e}$ as $b_{e} \rightarrow 0$ in the numerical calculations. We have assumed that the heavy charged lepton mass matrix $M_{E}$ is given by $M_{E}=\lambda m_{0}\left(\mathbf{1}+3 b_{e} X_{2}\right)$ on the basis of $F\left(\operatorname{not} F^{\prime}\right)$, i.e., $M_{E}$ has the partially democratic form. However, the choice $b_{e}=0$ makes this assumption nonsense. We consider that the value of the parameter $b_{e}$ is $b_{e} \simeq 0$, but it is not $b_{e}$ $=0$. In fact, although the relation (3.6) has given, for the observed charged lepton mass values $m_{e}$ and $m_{\mu}$, an excellent prediction of the tau lepton mass $m_{\tau}$, for the values [16] of $m_{e}$ and $m_{\mu}$ at $\mu=m_{Z}$ we obtain the predicted value $m_{\tau}\left(m_{Z}\right)=1724.99 \mathrm{MeV}$, which slightly deviates from the observed value $m_{\tau}\left(m_{Z}\right)=1746.69_{-0.27}^{+0.30} \mathrm{MeV}$ [16]. This deviation can be adjusted by taking a small deviation of $b_{e}$ from zero.

\section{MEANINGS OF THE ROTATION $\boldsymbol{R}_{X}$}

In the previous section, we found that the values of the parameters $x_{i}$ with the requirement $y_{1}=y_{3}$ are very close to the values of $z_{i}$, which are evaluated from the observed charged lepton masses. It should be noted that only for such a case with $x_{i} \simeq z_{i}$ do we obtain a solution for the value of the parameter $b_{\nu}$ that gives reasonable masses and mixings. In other words, even if we do not require the condition $y_{1}$ $=y_{3}$, the phenomenological two-parameter study with $\varepsilon$ and $b_{\nu}$ can find a reasonable solution only when $x_{i} \simeq z_{i}$. This suggests that the rotation $R_{X}$ has a special meaning not only for the neutrino mass matrix, but also for the charged lepton mass parameter matrix $Z$. We consider that the coincidence $x_{i} \simeq z_{i}$ is not accidental.

The rotation $R_{X}$ has the following property:

$$
R_{X}\left(\begin{array}{c}
x_{3} \\
x_{2} \\
x_{1}
\end{array}\right)=\left(\begin{array}{l}
1 \\
0 \\
0
\end{array}\right)
$$

in addition to the property (2.14). Therefore, it means that the parameters $z_{i}$ can be obtained from the vector $(1,0,0)$ by the following rotation: 


$$
\left(\begin{array}{c}
z_{3} \\
z_{2} \\
z_{1}
\end{array}\right)=\left(R_{X}\right)_{x_{i}=z_{i}}^{T}\left(\begin{array}{l}
1 \\
0 \\
0
\end{array}\right) .
$$

If we define a rotation matrix $\widetilde{R}_{X}$ as

$$
\widetilde{R}_{X}=T R_{X} T,
$$

where $T$ is defined by Eq. (2.18), the relations become more intuitive:

$$
\begin{gathered}
\widetilde{R}_{X} X_{3} \widetilde{R}_{X}^{T}=\widetilde{X}_{2} \equiv \frac{1}{2}\left(\begin{array}{ccc}
0 & 0 & 0 \\
0 & 1 & 1 \\
0 & 1 & 1
\end{array}\right), \\
\left(\widetilde{R}_{X}\right)_{x_{i}=z_{i}}\left(\begin{array}{c}
z_{1} \\
z_{2} \\
z_{3}
\end{array}\right)=\left(\begin{array}{l}
0 \\
0 \\
1
\end{array}\right), \\
\left(\widetilde{R}_{X}\right)_{x_{i}=z_{i}} \cdot Z \cdot\left(3 X_{3}\right) \cdot Z \cdot\left(\widetilde{R}_{X}\right)_{x_{i}=z_{i}}^{T}=\left(\begin{array}{lll}
0 & 0 & 0 \\
0 & 0 & 0 \\
0 & 0 & 1
\end{array}\right) .
\end{gathered}
$$

However, in order to obtain the same numerical results as those in the previous section, we must change the assumption $X_{\nu}=R_{X} P_{3} X_{3} P_{3} R_{X}^{T}$ to the following assumption:

$$
X_{\nu}=\widetilde{R}_{X} P_{1} X_{3} P_{1} \widetilde{R}_{X}^{T},
$$

where

$$
P_{1}=\operatorname{diag}(-1,1,1)
$$

Then the parameters $y_{i}$ in the expression (2.26) are given by the same relations with an exchange between $y_{1}$ and $y_{3}$. Since we require $y_{1}=y_{3}$, the numerical results are exactly identical with those in the previous section. In the previous scenario we assumed, with the rotation $R_{X}$, that the heavy up fermions take the same phase matrix $P_{3}=P\left(\delta_{u}\right)=P\left(\delta_{\nu}\right)$. In this case with $\widetilde{R}_{X}$, we must assume that $P\left(\delta_{u}\right)=P_{3}$, but $P\left(\delta_{\nu}\right)=P_{1}$. Although the scenario with $\widetilde{R}_{X}$ is more intuitive, we cannot at present answer the questions why quarks require the inversion $P_{3}$ and why leptons require the inversion $P_{1}$.

In any case, it is essential that the parameter values $\left(z_{1}, z_{2}, z_{3}\right)$ [or $\left.\left(z_{3}, z_{2}, z_{1}\right)\right]$ come from $(0,0,1)$ [or $\left.(1,0,0)\right]$ by the rotation $\widetilde{R}_{X}$ (or $R_{X}$ ). In particular, it is noted that the parameters $z_{i}$ satisfy the relation (2.23) [and therefore (3.7)], which leads to the charged lepton mass relation (3.6). Thus, the rotation $R_{X}$ has special meanings not only as a rotation between the heavy quarks $(U, D)$ and $(N, E)$, but also as a rotation that determines the charged lepton mass parameters $z_{i}$.

\section{CONCLUSIONS}

We proposed an improved version of the democratic universal seesaw model in order to extend the success of the unified description of the quark and charged lepton mass matrices to the neutrino mass matrix. In the original model, the mass matrices $m_{L}$ and $m_{R}$ were given by a universal structure $Z$, independently of the fermion sectors $f$ $=u, d, e, \nu$, and the hypothetical heavy fermion mass matrices $M_{F}$ have the same structure, "a unit matrix plus a democratic matrix," which includes only one flavor-dependent complex parameter $b_{f}$. The constraint was too tight, so that the model could not give the observed nearly bimaximal neutrino mixing. In the improved model, the mass matrices $m_{L}^{f}$ (also $m_{R}^{f}$ ) are still flavor independent, while the heavy fermion mass matrices have different structures between quark and lepton sectors, i.e., in the quark sectors, $M_{F}$ still have democratic forms, while in the lepton sector, $M_{F}$ have only "partially" democratic forms. If we take a special rotation $R_{X}$, which transforms the $3 \times 3$ democratic matrix $X_{3}$ to the $2 \times 2$ democratic matrix $X_{2}$ as Eq. (2.14) and if we take the parameters $x_{i}$ as $x_{i} \simeq z_{i} \propto \sqrt{m_{i}^{e}}$ and $b_{\nu} \simeq-2 / 3$, we can obtain reasonable values of the neutrino masses and mixings.

For the quark and charged lepton sectors, in the original democratic universal seesaw model [2,3], we already obtained reasonable values of the masses and mixings by taking $b_{e}=0, b_{u}=-1 / 3$, and $b_{d} \simeq-1$. Those values of $b_{f}$ are unchanged in the present revised model and, moreover, in order to explain the observed nearly bimaximal neutrino mixing, the value $b_{\nu} \simeq-2 / 3$ is required. What is the meaning of these parameter values

$$
b_{e}=0, \quad b_{u}=-1 / 3, \quad b_{\nu} \simeq-2 / 3, \quad b_{d} \simeq-1 ?
$$

This is a future task for us.

We also searched numerically for a rotation matrix $R\left(\theta_{12}, \theta_{23}, \theta_{31}\right)$ that can give reasonable values for the observed neutrino mixings and masses, without requiring the constraint (2.14). We found that the only solution is the rotation $R_{X}$ with $x_{i} \simeq z_{i}$ [the values $z_{i}$ are given by Eq. (1.11)] for $b_{\nu} \simeq-2 / 3$. The solution $R_{X}$ transforms the "fully" democratic matrix $X_{3}$ into the partially democratic matrix $X_{2}$ and the parameters $x_{i}$ satisfy the relation (3.5), which leads to the charged lepton mass formula (3.6). The rotation $R_{X}$ with $x_{i}$ $\simeq z_{i}$ also transforms the matrix $X_{3}^{\prime}$ (2.24) into a partially democratic matrix $X_{v}(2.25)$ with $y_{1}=y_{3}$. These results mean that the observed neutrino data require not a mere numerical solution of $R\left(\theta_{12}, \theta_{23}, \theta_{31}\right)$, but the special solution $R_{X}$ with $x_{i}=z_{i}$. The observed charged lepton masses, which are proportional to $z_{i}^{2}$, are closely related to the rotation $R_{X}$ with $x_{i}=z_{i}$, for example, as Eqs. (4.2), (3.7), and so on. These facts give us a sufficient motivation for the rotation $R_{X}$ with $x_{i}=z_{i}$ to be taken seriously. However, at present, the theoretical origin of the rotation is not clear. This is also a future task for us.

\section{ACKNOWLEDGMENTS}

The authors wish to acknowledge the hospitality of the theory group at CERN, where this work was completed. 
[1] Z. G. Berezhiani, Phys. Lett. 129B, 99 (1983); 150B, 177 (1985); D. Chang and R. N. Mohapatra, Phys. Rev. Lett. 58, 1600 (1987); A. Davidson and K. C. Wali, ibid. 59, 393 (1987); S. Rajpoot, Mod. Phys. Lett. A 2, 307 (1987); Phys. Lett. B 191, 122 (1987); Phys. Rev. D 36, 1479 (1987); K. B. Babu and R. N. Mohapatra, Phys. Rev. Lett. 62, 1079 (1989); Phys. Rev. D 41, 1286 (1990); S. Ranfone, ibid. 42, 3819 (1990); A. Davidson, S. Ranfone, and K. C. Wali, ibid. 41, 208 (1990); I. Sogami and T. Shinohara, Prog. Theor. Phys. 66, 1031 (1991); Phys. Rev. D 47, 2905 (1993); Z. G. Berezhiani and R. Rattazzi, Phys. Lett. B 279, 124 (1992); P. Cho, Phys. Rev. D 48, 5331 (1994); A. Davidson, L. Michel, M. L. Sage, and K. C. Wali, ibid. 49, 1378 (1994); W. A. Ponce, A. Zepeda, and R. G. Lozano, ibid. 49, 4954 (1994).

[2] Y. Koide and H. Fusaoka, Z. Phys. C 71, 459 (1996); Prog. Theor. Phys. 97, 459 (1997).

[3] Y. Koide and H. Fusaoka, Prog. Theor. Phys. 97, 459 (1997).

[4] T. Morozumi, T. Satou, M. N. Rebelo, and M. Tanimoto, Phys. Lett. B 410, 233 (1997).

[5] Y. Koide, Eur. Phys. J. C 9, 335 (1999); Phys. Rev. D 61, 035008 (2000).

[6] N. Cabibbo, Phys. Rev. Lett. 10, 531 (1963); M. Kobayashi and T. Maskawa, Prog. Theor. Phys. 49, 652 (1973).
[7] Y. Koide, Mod. Phys. Lett. A 22, 2071 (1993).

[8] Y. Koide and H. Fusaoka, Phys. Rev. D 64, 053014 (2001).

[9] Y. Koide, Mod. Phys. Lett. A 11, 2849 (1996); Phys. Rev. D 57, 5836 (1998).

[10] Super-Kamiokande Collaboration, Y. Fukuda et al., Phys. Rev. Lett. 81, 1562 (1998); M. Shiozawa, talk presented at "Neutrino 2002," Munich, Germany, 2002, http:// neutrino.t30.physik.tu-muenchen.de/

[11] Y. Suzuki, talk presented at "Neutrino 2000," Sudbury, Canada, 2000, http://nu2000.sno.laurentian.ca/; also see M. Gonzalez-Garcia, talk presented at "Neutrino 2000," Sudbury, Canada, 2000, http://nu2000.sno.laurentian.ca/

[12] SNO Collaboration, Q. R. Ahmad et al., Phys. Rev. Lett. 89, 011302 (2002).

[13] Z. Maki, M. Nakagawa, and S. Sakata, Prog. Theor. Phys. 28, 870 (1962); B. Pontecorvo, Zh. Eksp. Teor. Fiz. 33, 549 (1957); Sov. Phys. JETP 26, 984 (1968).

[14] Y. Koide, Lett. Nuovo Cimento Soc. Ital. Fis. 34, 201 (1982); Phys. Lett. 120B, 161 (1983); Phys. Rev. D 28, 252 (1983); also see, Y. Koide and M. Tanimoto, Z. Phys. C 72, 333 (1996); Y. Koide, Phys. Rev. D 60, 077301 (1999).

[15] Y. Koide, Phys. Rev. D 64, 077301 (2001).

[16] H. Fusaoka and Y. Koide, Phys. Rev. D 57, 3986 (1998). 